\title{
A SEARCH GAME FOR A MOBILE TARGET WITH THE CONDITIONALLY DETERMINISTIC MOTION DEFINED BY PATHS
}

\author{
Koji Iida Ryusuke Hohzaki Shingo Furui \\ The National Defense Academy
}

(Received December 13, 1994; Revised August 24, 1995)

\begin{abstract}
In this paper, we investigate a search-and-hide game played by a searcher and a mobile target with a conditionally deterministic motion. The target space consists of discrete cells and there are possible target's paths which specify the target's position (cell number) at all times in future. The target selects one of paths at the beginning of the game and moves along the path thereafter. The searcher knows all possible paths of the target but he cannot know which path is selected by the target. The searcher is restricted by his total searching effort at each time, and allocating it among cells, he searches the target. We formulate this search situation as a two-person zero-sum game and derive the optimal solution. Several examples are examined and the meaning of the optimal conditions are elucidated.
\end{abstract}

\section{Introduction}

In this paper, we deal with a two-sided search game played by a searcher and a mobile target with a rather simple type of motion called the conditionally deterministic motion (abbreviated as CDM, hereafter). The CDM target was dealt with by Stone $[5,6]$ first in his study of the optimal distribution of searching effort in the one-sided search. The CDI target is defined generally as a moving target whose motion takes place in Euclidean space and depends on an stochastic parameter such as the target's velocity and so on. If this parameter were known, then the target's position would be known at all times in future. However, the CDM target dealt with in this paper is more restrictive than the general one in a sense that the stochastic parameters are defined by target's paths on the target space. The target space consists of discrete $m$ cells and the time space also does discrete $n$ time points. A path $\omega$ of the target is defined as a sequence of cell numbers which specifies the target's position at each time. Set of possible paths of the target is known to the searcher. The target selects one of paths at the beginning of the game and moves along the path thereafter. On the other hand, a searcher is restricted by his available total searching effort at each time, and allocating it among cells, he searches cells for the target. Here, we assume that the searcher wants to detect the target and the target does not. In this paper, we formulate this search situation as a two-person zero-sum game and derive optimal strategies.

The two-sided search for a mobile target such as the well known problem of "the princess and monster game" posed by Isaacs and its variations, the search games on a graph or tree, the ambush search games and so on, have been investigated by many authors [1] and some results of them were compiled and published by Gal [2]. However, we cannot find any paper which gives the solution of the rather simple game dealt with here. As shown later, the payoff function of our game is presented by a strictly convex function of the searcher's strategy for every target's strategies. Hence, from the vicwpoint of theoretical study of the game theory, the existence of the optimal 
solution and its properties have been known. However, from the standpoint of the twosided search problems, our knowledge is very little. Furthermore, since the target's movement may be restricted in several paths and the set of possible paths is known by the searcher in many cases of actual search, our game may be valuable to analyze and be useful for practical applications.

In the next section, system parameters are defined and assumptions of the model are described in detail. In $\S 3$, the probiem is formulated and the optimal solution is presented. Several generalizations of our model are analyzed in $\S 4$ and numerical examples are examined in $\$ \overline{5}$. Finally in $\$ 6$, the implications of the optimal conditions and the results obtained here are discussed.

\section{Assumptions of the Model}

The definition of the system parameters and the assumptions of the model are presented as follows.

(1). The target space consists of $m$ discrete cells $j=1,2, \cdots, m$, and the target's movement and the search take place at discrete time points $t=1,2, \cdots, n$.

(2). The target's path is defined as a sequence of cell numbers $\omega=\{j(t), t=1,2, \cdots, n\}$ where $j(t)$ is the cell in which the target exists at time $t$. The path is permitted to stay on the same cell, to skip the cells, and to appear or vanish from the target space. The number $K$ of the possible paths is assumed to be finite.

(3). The target selects one of $K$ paths at the beginning of the game so as to avoid detection by the searcher and moves along the path thereafter. Let $T_{t}(\omega)$ be the cell of the target selecting the path $\omega$ at time $t$. In each time $t$, first the target moves to cell $T_{t}(\omega)$ from $T_{t-1}(\omega)$, and then the searcher searches cells for the target. We assume that if the target is not found in the time interval $[1, n]$, he gains a score 1 from the searcher, and if he is detected, he gives a score 1 to the searcher.

(4). Total searching effort $\{C(t), t=1,2, \cdots, n\}$ is available to the searcher, and the effort $C(t)$ is assumed to be continuously divisible in allocating it among cells. The searching effort allocated to cell $j$ at time $t$ is denoted by $\phi(j, t)$.

(5). If the target exists in cell $j$ at time $t$, the conditional probability of detecting the target with unit searching effort is assumed to be $\alpha(j)(>0)$ irrespective of the history of the past search. This assumption implies that the random search is conducted in each cell and the non-detection probability of the target with the searching effort $\phi(j, t)$ is given by

$$
f(j, \phi(j, t))=\exp (-\alpha(j) \phi(j, t)),
$$

which is called as the exponential detection function.

(6). The parameters: $\{C(t)\},\{\alpha(j)\}$ and $\{\omega\}$ are assumed to be known to both players in advance of the game.

(7). The target's payoff is defined by his score. Here, we assume that both the target and the searcher are opposing against each other completely in a sense that the target desires to maximize his score and the searcher wants to minimize it. The omniscient rationality of both players is assumed.

\section{Formulation of the Search Game and the 0ptimal Solution}

\subsection{Formulation of the game}

The target's pure strategy is defined by his selection of path $\omega$. On the other hand, the searcher's pure strategy is defined by the allocation of the searching 
effort: $\Phi=\{\dot{\phi}(j, t)\}$, where constraints: $\Sigma_{j} \phi(j, t) \leqq C(t)$ for all $t$ and $\phi(j, t) \geqq 0$ for all $j$ and $t$, are imposed on $\Phi$.

Let $g(\omega, \Phi)$ be the conditional non-detection probability of the target in the game when the strategies $\omega$ and $\Phi$ are employed by both players. We have

$$
g(\omega, \Phi)=\exp \left\{-\Sigma_{t} \alpha\left(T_{t}(\omega)\right) \phi\left(T_{t}(\omega), t\right)\right\} .
$$

Then, the expected score of the target is given by $\{2 g(\omega, \Phi)-1\}$ and in this value, the factor (2) in the first term and the second term $(-1)$ have not any effect on the optimization of the game. Therefore, we can define the payoff function of our game (the conditional expected score of the target) by $g(\omega, \Phi)$. Since $g(\omega, \Phi)$ is the exponential function of $\{\phi(j, t)\}$ for any $\omega$ by Eq. (2), the next lemma is obvious.

Lemma 1. $g(\omega, \Phi)$ is strictly convex in $\Phi$ for any $\omega$.

Since our search game is a strictly convex game by Lemma 1 , there exists a unique optimal solution. Here, we quote the basic theorem of the strictly convex game from a text book [7] without proof.

Lemma 2. In a strictly convex game, the minimizer (the searcher) possesses a unique optimal strategy; moreover, this strategy is pure.

We define the mixed strategy of the target by $P=\left\{p_{\omega}, \omega=1,2, \cdots, K\right\}$, where $p_{\omega}$ is the probability that the target selects path $\omega$. The expected payoff $G(P, \Phi)$ of the target is given by

$$
G(P, \Phi)=\Sigma_{\omega} p_{\omega} g(\omega, \Phi)=\Sigma_{\omega} p_{\omega} \exp \left\{-\Sigma_{t} \alpha\left(T_{t}(\omega)\right) \phi\left(T_{t}(\omega), t\right)\right\}
$$

Therefore, our problem is formulated as the game to obtain the optimal strategy $\left(P^{*}, \Phi^{*}\right)$ and the value of the game $G$ satisfying

$$
G\left(P^{*}, \Phi^{*}\right) \equiv G=\min _{\oplus} \max _{P} G(P, \Phi)=\max _{F} \min _{\diamond} G(P, \Phi),
$$

subject to constraints:

$$
\begin{aligned}
\sum_{j} \phi(j, t) \leqq & C(t) \text { for all } t \text { and } \phi(j, t) \geqq 0 \text { for all } t \text { and } j, \\
& \sum_{\omega} p_{\omega}=1 \text { and } p_{\omega} \geqq 0 \text { for all } \omega .
\end{aligned}
$$

\subsection{0ptimal solution of the game}

Let $P^{*}=\left\{p_{\omega}^{*}\right\}$ and $\Phi^{*}=\left\{\phi^{*}(j, t)\right\}$ be the ontimal strategies of the target and the searcher, respectively. The optimal solution of our game is presented by the next theorem.

Theorem 1. Under the constraint (4), we define $\mu_{0}$ by

$$
\mu_{0}=\max _{\omega} g\left(\omega, \Phi^{*}\right) \text {. }
$$

Then, the optimal strategy $\left\{p_{\omega}{ }^{*}\right\}$ of the target is given by

$$
\begin{aligned}
& \text { if } p_{\omega}{ }^{*}>0, \quad \exp \left(-\Sigma_{t} \alpha\left(T_{t}(\omega)\right) \phi^{*}\left(T_{t}(\omega), t\right)\right)=\mu_{0}, \\
& \text { if } p_{\omega}{ }^{*}=0, \quad \exp \left(-\Sigma_{t} \alpha\left(T_{t}(\omega)\right) \phi^{*}\left(T_{t}(\omega), t\right)\right) \leqq \mu_{0},
\end{aligned}
$$

for all $\omega$ and Eq. (5). On the other hand, the optimal strategy $\left\{\phi^{*}(j, t)\right\}$ of the searcher is given by Eq. (4) and

$$
\begin{aligned}
& \text { if } \phi^{*}(j, t)>0, \quad \alpha(j) \sum_{\omega \in \Omega(}(j, t), p_{\omega}^{*}=\frac{\lambda_{t}}{\mu_{0}}, \\
& \text { if } \phi^{*}(t, j)=0, \quad \alpha(j) \sum_{\omega \in \Omega(}(j, t) p_{\omega}^{*} \leqq \frac{\lambda_{t}}{\mu_{0}},
\end{aligned}
$$

for all $t$ and $j$, where $\Omega(j, t)$ is the set of the target's path such that $\Omega(j, t)=$ $\left\{\omega: T_{t}(\omega)=j\right\}$, and $\lambda_{t}$ is a non-negative Lagrange multiplier. 
The value of the game is given by

$$
G=\mu_{0} .
$$

Proof. As is well known in the game theory, $P^{*}=\left\{p_{\omega}{ }^{*}\right\}$ and $\Phi^{*}=\left\{\phi^{*}(j, t)\right\}$ are optimal if and only if

$$
G\left(P, \Phi^{*}\right) \leqq G \leqq G\left(P^{*}, \Phi\right) \text { for any } P \text { and } \Phi .
$$

Theorem is proved by showing the above. The first inequality of Eq. (10):G(P, $\left.\Phi^{*}\right) \leqq G$ is rewritten as $\max _{F} G\left(P, \Phi^{*}\right) \leqq G$. As seen in Eq. (3), $G\left(P, \Phi^{*}\right)$ is the linear function of $p_{\omega}$, and therefore, $\max _{P} G\left(P, \Phi^{*}\right)$ is given by

$$
\begin{aligned}
& \text { if } p_{\omega}>0, \exp \left\{-\Sigma_{t} \alpha\left(T_{t}(\omega)\right) \phi^{*}\left(T_{t}(\omega), t\right)\right\}=Z, \\
& \text { if } p_{\omega}=0, \exp \left\{-\Sigma_{t} \alpha\left(T_{t}(\omega)\right) \phi^{*}\left(T_{t}(\omega), t\right)\right\} \leqq Z,
\end{aligned}
$$

for $Z=\max _{\omega}\left\{\exp \left\{-\Sigma_{t} \alpha\left(T_{t}(\omega)\right) \phi^{*}\left(T_{t}(\omega), t\right)\right\}\right\}$ under the constraint $(5)$. Since $0<Z$ $\leqq 1$ obviously, we have $Z=\mu_{0}$, then Eqs. (6) and (7) are derived from the above. Eq. (9) is easily confirmed by substituting Ea. (7) into Eq. (3).

The second inequality of Eq. (10): $G \leqq G\left(P^{*}, \Phi\right)$ is the same problem that $\min _{\Phi} G\left(P^{*}, \Phi\right) \geqq G$ subject to Eq. (4). As easily seen from Eq. (3), $G\left(P^{*}, \Phi\right)$ is strictly convex in $\Phi$ and the constraint is a linear function from Eq. (4). Therefore, the necessary and sufficient condition for $\min _{\phi} G\left(P^{*}, \Phi\right)$ is obtained from the Kuhn-Tucker Theorem as :

$$
\begin{array}{ll}
\text { if } \phi^{*}(j, t)>0, & \frac{\partial G\left(P^{*}, \Phi\right)}{\partial \phi^{*}(j, t)}=\lambda_{t}, \\
\text { if } \phi^{*}(j, t)=0, & \frac{\partial G\left(P^{*}, \Phi\right)}{\partial \phi^{*}(j, t)} \leqq \lambda_{t},
\end{array}
$$

for a Lagrange multiplier $\lambda_{t}(\geqq 0)$ with $\lambda_{t}\left(C(t)-\Sigma_{j} \phi(j, t)\right)=0 . \partial G\left(P^{*}, \Phi\right) / \partial \phi^{*}(j, t)$ is calculated from Eq. (3). Substituting it and Eq. (7) into the above equation (11), we have Eq. (8). Eq. (9) is derived by substituting Eqs. (7) and (8) into Eq. (3).

The searcher's optimal strategy $\left\{\phi^{*}(j, t)\right\}$ and $\mu_{0}$ are obtained from Eqs. (4) and (6). The equation (6) is rewritten by another expression:

$$
\mu_{0}=\min _{\Phi}\left\{\mu \mid \Sigma_{i} \alpha\left(T_{t}(\omega)\right) \phi^{*}\left(T_{t}(\omega), t\right) \geqq-\log \mu \text { for all } \omega\right\},
$$

subject to the constraints (4). Since the problem given by the above equations can be converted to a linear programming problem, we can solve it by using the simplex method easily. Then, the optimal strategy $\left\{p_{\omega}{ }^{*}\right\}$ of the target is obtained from Eq. (8) and the value of the game is given by $\mu_{0}$.

\section{Generalization of the Mode1}

In this section, we discuss several generalization of our game.

\subsection{The model with the regular detection function}

In the model described in the previous section, we assume an exponential detection function given by Eq. (1). Without any difficulty, Theorem 1 can be generalized to more general detection function $f(j, \phi(j, t)$ ) so called the regular detection function having the next properties.

$$
\begin{array}{ll}
f(j, 0)=1, & f(j, \infty)=a(\geqq 0), \\
\frac{\partial f(j, \phi)}{\partial \phi}<0, & \frac{\partial^{2} f(j, \phi)}{\partial \phi^{2}}>0,
\end{array}
$$

for all $j$. The above properties mean that the searching effort is subject to the law 
of diminishing marginal return. Furthermore, we assume that the detection in any $j$ and $t$ is independent. In this case, the payoff $g$ and the expected payoff $G$ of the target are given by

$$
\begin{aligned}
& g(\omega, \Phi)=\Pi_{t} f\left(T_{t}(\omega), \phi\left(T_{t}(\omega), t\right)\right), \\
& G(P, \Phi)=\sum_{\omega} p_{\omega} \Pi_{t} f\left(T_{t}(\omega), \phi\left(T_{t}(\omega), t\right)\right),
\end{aligned}
$$

instead of Eqs. (2) and (3), respectively. In this case, generally the payoff $g$ given by Eq. (13) is not convex any more, except for the special $f$ such as the exponential detection function. And therefore, the game is not the convex game. However, we can prove that the search game has an optimal strategy $\left(P^{*}, \Phi^{*}\right)$ given by the next theorem.

Theorem 2. The optimal solution of the game with the regular detection function is stated as follows. Under the constraint (4), we define $u_{0}$ by

$$
\mu_{0}=\max _{\omega} g\left(\omega, \Phi^{*}\right) \text {. }
$$

Then, the optimal strategy $\left\{p_{\omega}{ }^{*}\right\}$ of the target is given by Eq. (5) and

$$
\begin{aligned}
& \text { if } p_{\omega}{ }^{*}>0, \quad \Pi_{t} f\left(T_{t}(\omega), \phi^{*}\left(T_{t}(\omega), t\right)\right)=\mu_{s} \text {, } \\
& \text { if } p_{\omega}{ }^{*}=0, \quad \Pi_{t} f\left(T_{t}(\omega), \phi^{*}\left(T_{t}(\omega), t\right)\right) \leqq \mu_{0} \text {, }
\end{aligned}
$$

for all $\omega$. The optimal strategy $\left\{\phi^{*}(j, t)\right\}$. of the searcher is given by Eq. (4) and

$$
\begin{aligned}
& \text { if } \phi^{*}(j, t)>0, \quad-\frac{f^{\prime}\left(j, \phi^{*}\left(T_{t}(\omega), t\right)\right)}{f\left(j, \phi^{*}\left(T_{t}(\omega), t\right)\right)} \sum_{\omega \in \Omega(j, t)} p_{\omega}{ }^{*}=\frac{\lambda_{t}}{\mu_{0}}, \\
& \text { if } \phi^{*}(j, t)=0, \quad-\frac{f^{\prime}\left(j, \phi^{*}\left(T_{t}(\omega), t\right)\right)}{f\left(j, \phi^{*}\left(T_{t}(\omega), t\right)\right)} \sum_{\omega \in \Omega(j, t)} p_{\omega}{ }^{*} \leqq \frac{\lambda_{t}}{\mu_{0}},
\end{aligned}
$$

for all $t$ and $j$, where $f^{\prime}(j, \phi)$ is the derivative $f$ with respect to $\phi$, and $\lambda_{t}$ is a non-negative Lagrange multiplier. The value of the game is given by

$$
G=\mu_{0} \text {. }
$$

Proof. By the similar manner to the proof of Theorem 1, Theorem 2 is also proved by conf irming Eq. (10). Since $G\left(P, \Phi^{*}\right)$ given by Eq. (14) is a linear function of $p_{\omega}$, Eqs. (15) and (16) are derived from the first inequality of (10). The second inequality of Eq. (10), equivalently, $\min _{\Phi} G\left(P^{*}, \Phi\right) \geqq G$ subject to Eq. (4), is proved by using the Kuhn-Tucker Theorem and Eq. (17) is derived for a necessary condition since $f(j, \phi)$ is strictly convex of $\phi$ by the assumption of Eq. (12). Eq. (18) is easily obtained by substituting Eqs. (15), (16) and (17) into Eq. (14).

By setting $f(j, \phi)=\exp (-\alpha(j) \phi(j, t))$, we can easily derive Theorem 1 from Theorem 2.

\subsection{The model with generalized searching effort constraints}

In Theorem 1, we consider the problem in which the total searching effort available to the searcher is limited by $c(t)$ in each time $t, t=1,2, \cdots, n$. However, in search problems for a moving target, generally the next three types of constraints for the searching effort can be considered.

$$
\begin{aligned}
& \Psi_{1}: \phi(j, t) \leqq B(j, t) \text { for all } t \text { and } j, \\
& \Psi_{2}: \Sigma_{j} \phi(j, t) \leqq C(t) \text { for all } t, \\
& \Psi_{3}: \Sigma_{t} \Sigma_{j} \phi(j, t) \leqq D .
\end{aligned}
$$

Condition $\Psi_{1}$ is the constraint of the effort in cell $j$ at time $t$ and Condition $\Psi_{2}$ is the constraint of the effort applied to the whole search space at $t$, and Condition 
$\Psi_{3}$ is the constraint of the total searching effort during the search. For convenience of notation, let $M\left(\Psi_{1}, \Psi_{2}, \Psi_{3}\right)$ be the problem imposed by these constraints and if the constraint $\Psi_{i}$ is not imposed, we denote it by $\Psi_{i}=\infty$. Then, the problems analyzed in the previous sections are denoted by $M\left(\infty, \Psi_{2}, \infty\right)$. In this section, we consider the problem $M\left(\Psi_{1}, \Psi_{2}, \Psi_{3}\right)$. Assuming the exponential detection function, we obtain the optimal solution of the game $M\left(\Psi_{1}, \Psi_{2}, \Psi_{3}\right)$ as follows.

Theorem 3. The optimal solution of the game with the constraint of searching effort $\left(\Psi_{1}, \Psi_{2}, \Psi_{3}\right)$ and with the exponential detection function is given by the following Eqs. (22) through (26). Under the constraint (4), we define $\mu_{0}$ by

$$
\mu_{0}=\max _{\omega} g\left(\omega, \Phi^{*}\right),
$$

where $g(\omega, \Phi)$ is defined by Eq. (2). Then, the optimal strategy $\left\{p_{\omega}{ }^{*}\right\}$ of the target is obtained by Eq. (5) and

$$
\begin{aligned}
& \text { if } p_{\omega}{ }^{*}>0, \quad \exp \left(-\Sigma_{t} \alpha\left(T_{t}(\omega)\right) \phi^{*}\left(T_{t}(\omega), t\right)\right)=\mu_{0}, \\
& \text { if } p_{\omega}{ }^{*}=0, \quad \exp \left(-\Sigma_{t} \alpha\left(T_{t}(\omega)\right) \phi^{*}\left(T_{t}(\omega), t\right)\right) \leqq \mu_{0},
\end{aligned}
$$

for all $\omega$. The optimal strategy $\left\{\phi^{*}(j, t)\right\}$ of the searcher is given by Eq. (4) and

$$
\begin{aligned}
& \text { if } \dot{\phi}^{*}(j, t)>0, \quad \alpha(j) \sum_{\omega \in \Omega(j, t)} p_{\omega}{ }^{*}=\frac{\lambda_{j t}+\lambda_{t}+\lambda_{0}}{\mu_{0}}, \\
& \text { if } \phi^{*}(j, t)=0, \quad \alpha(j) \sum_{\omega \in \Omega(j, t)} p_{\omega}{ }^{*} \leqq \frac{\lambda_{j}+\lambda_{t}+\lambda_{0}}{\mu_{0}},
\end{aligned}
$$

for all $t$ and $j$, where $\lambda_{j t}, \lambda_{t}$ and $\lambda_{0}$ are non-negative Lagrange multipliers corresponding to the searching effort constraints $\Psi_{1}, \Psi_{2}$ and $\Psi_{3}$, respectively. The value of these multipliers are

$$
\begin{aligned}
\text { as for } \lambda_{j t}: & \text { if } \Psi_{1}=\infty, \lambda_{j t}=0, \\
& \text { if } \Psi_{1} \neq \infty \text { and } \phi^{*}(j, t)<B(j, t), \quad \lambda_{j t}=0, \\
& \text { if } \Psi_{1} \neq \infty \text { and } \phi^{*}(j, t)=B(j, t), \quad \lambda_{j t} \geqq 0, \\
\text { as for } \lambda_{t}: & \text { if } \Psi_{2}=\infty, \lambda_{t}=0, \\
& \text { if } \Psi_{2} \neq \infty \text { and } \Sigma_{j} \phi(j, t)<C(t), \quad \lambda_{t}=0, \\
& \text { if } \Psi_{2} \neq \infty \text { and } \Sigma_{j} \phi(j, t)=C(t), \quad \lambda_{t} \geqq 0, \\
\text { as for } \lambda_{0}: \text { if } \Psi_{3}=\infty, \lambda_{0}=0, & \\
& \text { if } \Psi_{3} \neq \infty \text { and } \Sigma_{t} \Sigma_{j} \phi(j, t)<D, \quad \lambda_{0}=0, \\
& \text { if } \Psi_{3} \neq \infty \text { and } \Sigma_{t} \Sigma_{j} \phi(j, t)=D, \quad \lambda_{0} \geqq 0 .
\end{aligned}
$$

The value of the game is given by

$$
G=\mu_{0} .
$$

Proof. Since the strictly convexity of $g$ in $\Phi$ for all $\omega$ is not affected by the constraints $\Psi_{1}, \Psi_{2}$ and $\Psi_{3}$, our game is still the convex game and Theorem is proved by similar manner to Theorem 1 . However, since the Lagrangian function is defined including three constraints $\Psi_{1}, \Psi_{2}$ and $\Psi_{3}$, the right-hand side of Eq. (11) given by Kuhn-Tucker's Theorem contains three Lagrange multipliers $\lambda_{t j}, \lambda_{t}$ and $\lambda_{0}$ instead of $\lambda_{t}$ in Eq. (11) and Eq. (24) is derived. Eq. (25) is obtained from the equations of the complementary slackness.

Theorem 3 is easily generalized to the model with the regular detection function defined by Eq. (12).

\section{Numerical Examples}

In this section, to see the properties of the solution of the search game, several numerical examples are analyzed varying the searching effort constraint. 
Case 1 . We consider a simple game with 3 cells $(m=3), 3$ time points $(n=3)$ and 4 paths $(K=4): \omega_{1}=(1,1,1), \omega_{2}=(1,2,2), \omega_{3}=(2,2,1), \omega_{4}=(3,2,2)$. Fig. 1 shows the four possible paths in $(j, t)$ space. The exponential detection function with $\alpha(j)=1$ for all $j$ is assumed. The searcher's total searching effort is assumed to be limited by $C(1)=0.9, C(2)=0.3$ and $C(3)=0.6$. From these system parameters, the constraint (4) and Eq. (6) are written down to a linear programming problem as stated before. This LP problem is solved by the simplex method and we have $\mu_{0}=0.427$ and the searcher's optimal strategy $\phi^{*}(j, t)$. Then, the simultaneous linear equations for $p_{\omega}{ }^{*}$ given by Eq. (8) are solved. The optimal solution is shown in Table 1.

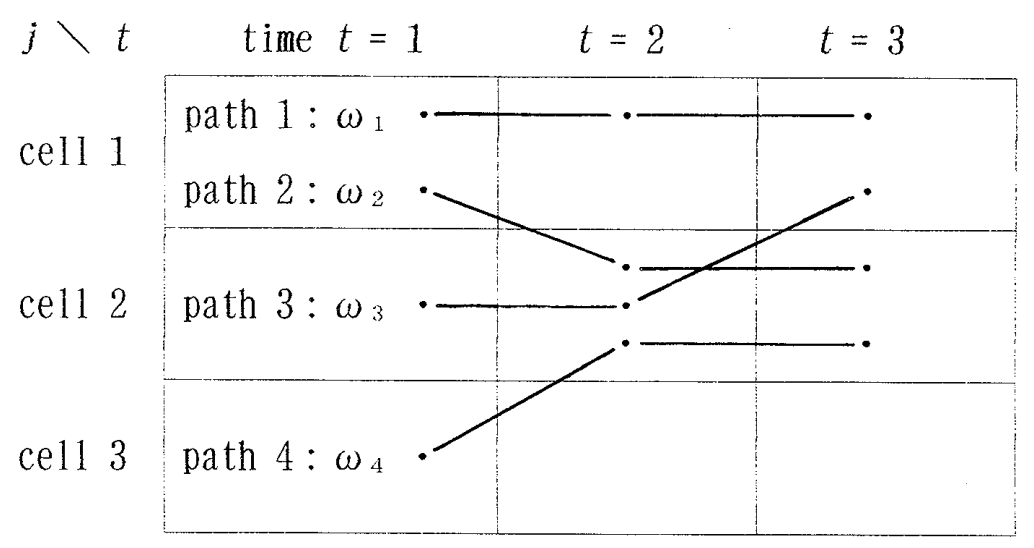

Fig. 1 Paths in $(j, t)$ space

Table 1 The optimal solution of Case 1

\begin{tabular}{|r|c|c|c|c|c|}
\hline$\Psi_{2}$ & $t$ & 1 & 2 & 3 & \multirow{2}{*}{$G$} \\
\cline { 1 - 5 } & $C(t)$ & 0.90 & 0.30 & 0.60 & $\left(\mu_{0}\right)$ \\
\hline \multirow{2}{*}{$\phi^{*}(j, t)$} & $j=1$ & 0.40 & 0 & 0.45 & \\
\cline { 1 - 5 } & 2 & 0.10 & 0.30 & 0.15 & \multirow{2}{*}{0.427} \\
\cline { 1 - 5 } & 3 & 0.40 & 0 & 0 & \multirow{2}{*}{$p_{\omega}^{*}$} \\
\cline { 1 - 5 } & $\omega_{1}$ & $\omega_{2}$ & $\omega_{3}$ & $\omega_{4}$ & \\
\cline { 1 - 4 } & $1 / 6$ & $1 / 6$ & $1 / 3$ & $1 / 3$ & \\
\hline
\end{tabular}

From Table 1, we can see the distinctive feature of the searcher's optimal strategy $\phi^{*}(j, t)$ : he concentrates his searching effort to the cross points of the target paths, and furthermore, he keeps the balance of allocation of the searching effort to each target's path. As seen in Fig. 1, the paths $\omega_{2}, \omega_{3}$ and $\omega_{4}$ intersect on cell 2 at time 2, and therefore, the searcher allocates all his searching effort $C(2)=0.3$ to this ce11 at time 2. On the other hand, we can confirm that $g(\omega, \Phi)$ is balanced to 0.427 for all $\omega$ and the searching effort allocated to each possible paths during the search is balanced to 0.85 . (It should be noted that $0.427=\exp (-0.85)$.) As for the target's optimal strategy $p_{\omega}{ }^{*}$, since the searcher searches all paths with same searching effort 0.85 , the target does not have any preferable path to avoid the detection. However, by selecting his path with $\left\{p_{\omega}{ }^{*}\right\}$, the target does not give any efficient cells to the searcher. 
Case 2. Case 2 is set by interchanging the total searching effort $c(t)^{\text {'s }} \mathrm{s}$ at $t=1$ and 2 in Case 1, namely, $C(1)=0.3, C(2)=0.9, C(3)=0.6$. Since the target's paths have many intersection in order of $t=2,3,1$, and therefore, the sequence of $C(t)$ is reasonable in this case. 0ther parameters remain the same as Case 1.

Table 2 shows an optimal solution of Case 2. In this case, the LP problem given by Eqs. (4) and (6) has the optimal value $\mu_{0}=0.407$ and the searching effort is concentrated to a cell at each time, and the effort to each target's path is not balanced : 0.9 for $\omega_{1}, 1.2$ for $\omega_{2}, 1.5$ for $\omega_{3}$ and 0.9 for $\omega_{4}$, where $0.407=$ $\exp (-0.9)$. Therefore, the target should not select $\omega_{2}$ and $\omega_{3}$.

Table 2 An optimal solution of Case 2

\begin{tabular}{|c|c|c|c|c|c|}
\hline & $t$ & 1 & 2 & 3 & \multirow{2}{*}{$G$} \\
\cline { 1 - 6 }$\Psi_{2}$ & $c(t)$ & 0.30 & 0.90 & 0.60 & \\
\hline & $j=1$ & 0.30 & 0 & 0.60 & \\
\multirow{2}{*}{$\phi^{*}(j, t)$} & 2 & 0 & 0.90 & 0 & \\
& 3 & 0 & 0 & 0 & \multirow{2}{*}{0.407} \\
\cline { 1 - 5 }$p_{\omega}^{*}$ & $\omega_{1}$ & $\omega_{2}$ & $\omega_{3}$ & $\omega_{4}$ & \\
\hline & 0.5 & 0 & 0 & 0.5 & \\
\hline
\end{tabular}

In Case 2, the LP problem has another extremal point as the optimal solution with the same value $\mu_{0}=0.407$ as shown in Table 3 . In Table 3 , the searching effort to each path is balanced to 0.90 . A11 $\phi(j, t)$ given by the linear combination of those $\phi^{*}(j, t)^{\prime}$ s given by Tables 2 and 3 are also optimal, and since the searching effort to $\omega_{2}$ and $\omega_{3}$ is larger than 0.9 in this case, the target should not select $\omega_{2}$ and $\omega_{3}$ too. It should be noted that the value of the game $G$ (the non-detection probability of the target) of Case 2 decreases compare with the value of Case 1 , in spite of the total searching effort during the search being constant 1.8. The reason is that since the target's paths at $t=2$ has more intersections than that at $t=1$, the increase of effort at $t=2$ brings on the decrease of the non-detection probability of the target. It suggests the optimal distribution of $\{C(t)\}$.

Table 3 Another optimal solution of Case 2

\begin{tabular}{|c|c|c|c|c|c|}
\hline & $t$ & 1 & 2 & 3 & $G$ \\
\cline { 1 - 5 }$\Psi_{2}$ & $c(t)$ & 0.30 & 0.90 & 0.60 & $\left(\mu_{0}\right)$ \\
\hline & $j=1$ & 0.15 & 0.375 & 0.375 & \\
\cline { 1 - 5 }$\phi^{*}(j, t)$ & 2 & 0 & 0.525 & 0.225 & \multirow{2}{*}{0.407} \\
\cline { 1 - 5 } & 3 & 0.15 & 0 & 0 & \multirow{2}{*}{$p_{\omega}^{*}$} \\
\cline { 1 - 5 } & $\omega_{1}$ & $\omega_{2}$ & $\omega_{3}$ & $\omega_{4}$ & \\
\cline { 1 - 5 } & 0.5 & 0 & 0 & 0.5 & \\
\hline
\end{tabular}


Case 3. Case 3 is an example of Theorem 3 . In this case, we consider a game with constraints: $M\left(\infty, \Psi_{2}, \Psi_{3}\right)$. Suppose that the searching effort is not limited in each cell $\left(\Psi_{1}=\infty\right)$, the total searching effort at each time is constrained by $\Psi_{2}$ : $\{C(1)=0.9, C(2)=0.3, C(3)=0.6\}$ (same as Case 1), and furthermore, the total searching effort during the search is 1 imited by $\Psi_{3}: D=1.5$. Since $\Psi_{3}$ is severer than $\Psi_{2}$ $\left(\Sigma_{t} C(t)=1.8\right)$, the searcher cannot exhaust all $C(t)$. The other parameters of the game are assumed to be the same as Case 1 . The optimal solution of this case is obtained as Table 4. In this table, the properties of $\phi^{*}(j, t)$ stated about Case 1 are also seen, since the arrangement of $C(t)$ is same as Case 1 . However, since $\Psi_{3}$ : $D=\infty$ in Case 1 , Case 3 is constrained by the total searching effort severer than Case 1 , and the effect appears on the value $\mu_{0}: \mu_{0}$ is increased from 0.427 in Case 1 to 0.472 in Case 3. It should be noted that the optimal distribution of total searching effort at each $t$ under the constraints $\Psi_{2}$ and $\Psi_{3}$ is $(0.6,0.3,0.6)$ as shown in Table 4. The searching effort at $t=1$ is smaller than the limit $C(1)$. This result seems to be reasonable since the concentration of the target's paths is fewest at $t=1$ as mentioned before. As for the optimal strategy $\left\{p_{\omega}{ }^{*}\right\}$ of the target, $\left\{p_{\omega}{ }^{*}\right\}$ is not determined uniquely in this case, because Eqs. (8) and (5) has only three equations and two inequalities for four unknown $p_{\omega}{ }^{*}$ 's. It is interesting that the feature of $p_{\omega}{ }^{*}$ in Cases 1 and 2 is also seen in Case 3.

Table 4 The optimal solution of Case 3

\begin{tabular}{|c|c|c|c|c|c|}
\hline & $t$ & 1 & 2 & 3 & \multirow{3}{*}{$\begin{array}{c}G \\
\left(\mu_{0}\right)\end{array}$} \\
\hline$\Psi_{2}$ & $C(t)$ & 0.90 & 0.30 & 0.60 & \\
\hline$\Psi_{3}$ & $D$ & \multicolumn{3}{|c|}{1.5} & \\
\hline \multirow{3}{*}{$\phi^{*}(j, t)$} & $j=1$ & 0.30 & 0 & 0.45 & \multirow{4}{*}{$\begin{array}{c}0.472 \\
\mu_{0}=0.75\end{array}$} \\
\hline & 2 & 0 & 0.30 & 0.15 & \\
\hline & 3 & 0.30 & 0 & 0 & \\
\hline \multirow{2}{*}{$p_{\omega}{ }^{*}$} & $\omega_{1}$ & $\omega_{2}$ & $\omega_{3}$ & $\omega_{4}$ & \\
\hline & $0.5-2 a$ & $a$ & $2 a$ & $0.5-a$ & $0 \leqq a \leqq 1 / 6$ \\
\hline
\end{tabular}

\section{Discussions}

In this section, we give the interpretation of the optimal conditions and discuss the results obtained in this paper and open problems to be studied in future.

1. Meaning of the optimal conditions

To consider the meaning of the optimal conditions, we examine Theorem 2 . The interpretations of the conditions of Theorem 2 from the view point of the searcher are described as follows. First, we consider the meaning of Eqs. (15) and (16). The term $\Pi_{t} f\left(T_{t}(\omega), \phi\left(T_{t}(\omega), t\right)\right)$ in these equations is the conditional non-detection probability of the target during the search given the target's path $\omega$. Hence, Eqs. (15) and (16) mean that the searcher should allocate his searching effort so as to balance this non-detection probability as small as possible to $\mu_{0}$. By this allocation, the searcher does not give any advantageous path to the target. Secondly, to explain the meaning of Eq. (17), let $P(j, t, \omega)$ be the detection probability of the target selecting path $\omega$ in cell $j$ at time $t$ during the search. Then, we have the next equation. 


$$
P(j, t, \omega) \doteq(1-f(j, \phi(j, t))) \prod_{s \neq t} f\left(T_{s}(\omega), \phi\left(T_{s}(\omega), s\right)\right) .
$$

By using the above, Eq. (17) is rewritten as

$$
\begin{aligned}
& \text { if } \phi^{*}(j, t)>0, \quad \sum_{\omega \in \Omega(j, t)} p_{\omega}{ }^{*} \frac{\partial P(j, t, \omega)}{\partial \phi(j, t)}=\lambda_{t}, \\
& \text { if } \phi^{*}(j, t)=0,\left.\quad \sum_{\omega \in \Omega(j, t)} p_{\omega}{ }^{*} \frac{\partial P(j, t, \omega)}{\partial \phi(j, t)}\right|_{\phi(j, t)=0} \leqq \lambda_{t} .
\end{aligned}
$$

Since the term $\sum_{\omega \in \Omega(2(j, t)} p_{\omega}{ }^{*} \partial P(j, t, \omega) / \partial \phi(j, t)$ means the marginal detection probability of the target at $(j, t)$, Eq. (17) is described as follows. If the searcher searches cell $j$ at time $t$, he should determine the searching effort so as to balance the marginal detection probability to $\lambda_{t}$ among cells searched at $t$, and if the the marginal detection probability at $\phi(j, t)=0$ is not larger than $\lambda_{t}$, cell $j$ must not be searched at $t$. By the theorem of the optimal search in the one-sided search for a moving target given by Iida [3], this allocation maximizes the overall detection probability of the target during the search.

on the other hand, from the viewpoint of the target, the optimal conditions of Theorem 2 are interpreted as follows. Eq. (16) means that the target should not select the path having smaller non-detection probability than $\mu_{0}$. Since these paths are dangerous for the target, this selection rule is reasonable. However, as stated above, the non-detection probability of the target on each path are minimized as small as possible by the searcher, the target does not have any preferable path. But by setting his path probability by Eq. (17), the target can avoid to give the searcher any efficient cell having the high marginal detection probability, and this strategy is effective for the target to maximize his payoff. The meaning of Theorem 2 mentioned above is completely valid to Theorems 1 and 3 , too.

2. Discussions on the Results

(1). In our model, if each path is defined by the same cell: $\omega=\{j(t)=i$ for all $t\}$, it implies that the target is stationary and then $\left\{p_{\omega}\right\}$ is the probability distribution of the target on the target space. Theorems 1,2 and 3 are also valid for this case. In this case, Theorem 1 is completely identical with the optimal solution of the search-and-hide game studied by Nakai [4] for an immobile target.

(2). In our model, the target's path is permitted in defect of terms in some time interval. Therefore, we can deal with the search game for the appearing and/or the disappearing target and Theorems 1,2 and 3 are all valid for these cases.

(3). As stated in $\$ 1$, we limit our CDM target to the case in which the parameter of the target's motion is defined by his path on the search space. In this case, we need not transform the searching on the search space into the parameter space. Our model may be easily generalized to the general CDM target defined by Stone [6] and the similar theorems as derived here may be also obtained. However, as stated by Stone, to derive clear results, we must assume that the absolute value of the Jacobian of the transformation is factorable in the parameter factor and the time factor. This assumption may limit the applicability of the model to the complex actual search situations. Since our search model of the mobile target defined by path is not troubled by the transformation, we can apply Theorems to considerably complex cases such that the target's paths having many cross points, the search for the appearing and/or the disappearing target, the case of time dependent constraints of the searching effort, etc. The generalization of our model to the general CDM target may have little merit from the viewpoint of applications.

(4). Several problems are remained to be investigated in future. In our search model, assuming the continuous searching effort, we define the searcher's strategy by the 
distribution of his searching effort. In this formulation, the kinematics of the searcher's movement is neglected. However, for the vehicular searcher, we must consider searcher's paths. Moreover, other generalizations of the model with respect to the following factors must be investigated.

(a). The model with other criterion such as the expected risk or the cost,

(b). he model of continuous target space,

(c). the model of continuous time space,

(d). the discrete searching effort case,

(e). the multitarget case,

(f). the multistage search game: the search-and-evasion game, etc.

Some of the above are easy, however, most of them may be very difficult. They are problems to be investigated in future.

\section{Acknowledgments}

The authors are very grateful to the referees for giving useful comments to refine this paper.

\section{References}

[1] Benkoski, S. J., Monticino, M. G. and Weisinger, J. R., "A Survey of the Search Theory Literature," Naval Research Logistics, 18 (1991), pp.469-494.

[2] Gal, S. : Search Games. Academic Press, 1980.

[3] Iida, K.: Studies on the 0ptimal Search Plan. Springer-Verlag, Berlin, 1992.

[4] Nakai, T., "Search Models with Continuous Effort under Various Criteria," J. Opns. Res. Soc. Jpn., 31 (1988), pp. 335-352.

[5] Stone, L.D. and Richardson, H. R., "Search for Targets with Conditionally Deterministic Motion," SIAM. J. App1. Math. 27 (1974), pp.239-255.

[6] Stone, L.D., "Search for Targets with Generalized Conditionally Deterministic Motion," SIAM. J.App1. Math. 33 (1977), pp. 456-468.

[7] Vorob' ev, N. N. : Game Theory. Springer-Verlag, 1977.

Koji IIDA : Department of Applied Physics, National Defense Academy, Hashirimizu 1-10-20, Yokosuka, 239, Japan. 